\title{
Autofluoreszenzbronchoskopie - schon bald Routine? - Contra
}

Eine kritische Würdigung

\section{B. Schmidt \\ C. Witt}

\section{Autofluorescence Bronchoscopy - Ready for Routine? - Contra. \\ A Critical Appreciation}

Optimismus ist nur Mangel an Information!

(Heiner Müller, Berlin 1929-1995)

Neue Entwicklungen folgen einem Schema: Zu Beginn finden sie das Interesse weniger Spezialisten. Sind sie spannend, folgt die Euphorie der großen Masse. Diese Euphorie wird von der Ernüchterung abgelöst, wenn erste Enttäuschungen auftreten, Nur bei tragfähigen Entwicklungen folgt die Phase einer Konsolidierung, einer realistischen, gut evaluierten Nutzung.

Hat die Autofluoreszenzbronchoskopie (AFB) diese letzte Phase erreicht?

Die Euphorie hat zu einer Flut von Veröffentlichungen zum Thema geführt, allein für das LIFE-System (Xillix, USA) gibt die Herstellerfirma mehr als 130 Publikationen an, meist kleinere Erfahrungsberichte. Für die neueren Geräte D-LIGHT (Storz, Germany), SAFE (Pentax, Japan) und DAFE (Wolff, Germany) ist die Zahl veröffentlichter Ergebnisse deutlich geringer. Analysiert man die vorliegenden Daten, so stellt man fest, dass die Zahl größerer Studien, die konventionelle Weißlicht- (WLB) und Autofluoreszenzbronchoskopie vergleichen, gering ist [1-6].

Lässt sich die Investition von 30000 bis 100000 Euro durch die vorliegenden Daten zum routinemäßigen Einsatz dieser Technik rechtfertigen? Im Folgenden soll durch kritische Fragen der Blick geschärft werden.

\section{Wie fundiert sind die Daten?}

In der Mehrzahl dieser Studien wurden Untersuchungen mit Weißlicht- und Autofluoreszenzbronchoskopie nacheinander durchgeführt. Eine Trennung der Informationen, die durch Weißlicht alleine oder durch die Autofluoreszenz gewonnen wird, ist bei diesem Ablauf unmöglich. Die klinische Erfahrung zeigt, dass manche AFB-positive (Autofluoreszenzbronchoskopie-positive) Läsionen anschließend auch im Weißlicht wiedererkannt werden können. Die AFB schärft den Blick für diskrete Veränderungen der Schleimhaut. Bisher liegt erst eine zweiarmige Studie zur Bewertung der Autofluoreszenzbronchoskopie vor. Daten aus dieser multizentrischen, randomisierten europäischen Studie wurden erstmalig 2002 auf dem World Congress for Bronchology 2002 in Boston [7] und umfassender auf dem Kongress der European Respiratory Society (ERS) 2003 in Wien präsentiert [8].

Die Autofluoreszenztechnik wurde in den meisten Studien mit konventionellen Fiberbronchoskopen verglichen und nicht mit Videobronchoskopen, die eine bessere Bildqualität liefern. Es ist zu erwarten, dass der Vorteil der AFB gegenüber der Videobronchoskopie geringer ist als gegenüber der konventionellen Fiberendoskopie.

Institutsangaben

Charité - Universitätsmedizin Berlin, Medizinische Klinik m.S. Kardiologie, Pneumologie,

Angiologie, Schwerpunkt Pneumologie, Campus Charité-Mitte, Berlin

Anmerkung

Die Autoren beteiligten sich an der Multizenterstudie: Autofluorescence bronchoscopy with white-light bronchoscopy compared to white-light bronchoscopy alone for the detection of precancerous lesions:

A European randomized controlled multicenter trial.

Diese Studie wurde von der Fa. Karl Storz GmbH \& Co., Tuttlingen unterstützt.

Korrespondenzadresse

Dr. med. Bernd Schmidt • Medizinische Klinik m. S. Kardiologie, Pneumologie, Angiologie .

Schwerpunkt Pneumologie (Prof. Dr. C. Witt) . Charité - Universitätsmedizin Berlin .

Campus Charité-Mitte·Schumannstr. 20-21 · 10098 Berlin·E-mail: b.schmidt@charite.de

Bibliografie

Pneumologie 2004; 58: 648-650 @ Georg Thieme Verlag KG Stuttgart · New York

DOI $10.1055 / \mathrm{s}-2004-830046$

ISSN 0934-8387 
Die detaillierten Angaben zu Biopsie-Strategien fehlen in fast allen Studien. Dabei werden die Daten zu Sensitivität und Spezifität durch die Strategie der Biopsie wesentlich beeinflusst. Die Vorgaben zu Kontrollbiopsien, sog. „random biopsies“, sind oft unpräzise. Eine Ursache für die hohe Inzidenz von Carcinomata in situ in einigen Studien (s.u.) könnte dadurch bedingt sein, dass Biopsien aus unmittelbarer Nachbarschaft eines invasiven Tumors entnommen wurden.

\section{In welchem Bereich ist nach den publizierten Daten} ein Vorteil der AFB gegenüber der WLB zu erwarten?

In den frühen Studien, die eine große Überlegenheit der Autofluoreszenzbronchoskopie zeigten, wurde eine vergleichsweise geringe Sensitivität der WLB bezüglich der Detektion invasiver Karzinome 26/40 (65\%) gefunden [4]. Die entsprechende Steigerung der Sensitivität auf 38/40 (95\%) durch den Einsatz der AFB wurde dieser Technik zugeschrieben. Dieser diagnostische Zugewinn konnte in späteren Studien nicht wieder gezeigt werden. Die nachfolgenden Untersuchungen zeigten identische Detektionsraten für invasive Karzinome bei WLB und AFB [2]. MorotSibilot u. Mitarb. fanden eine Sensitivität von 74,4 Prozent mit Weißlicht bzw. 77 Prozent mit Autofluoreszenz bei der Untersuchung von 244 Patienten [3]. Der Vorteil der AFB gegenüber der WLB besteht also in der verbesserten Detektion von Dysplasien und Carcinomata in situ.

Wie ist die Bedeutung von Dysplasie und Carcinoma in situ bezüglich einer Tumor-Früherkennung einzuschätzen?

Das Carcinoma in situ ist selten. In den Studien zur AFB wird eine Inzidenz zwischen 0 und $14 \%$ berichtet (Tab.1). Bei 54 Risikopatienten fanden Homasson u. Mitarb. 18 invasive Karzinome aber kein Cis [9]. Kusunoki u. Mitarb. fanden bei 9 von 65 Patienten mit Verdacht auf Bronchialkarzinom ein Cis [10]. In der multizentrischen europäische Studie an 1173 Patienten lag die Inzidenz bei $0,8 \%$ [7]. Dabei wurden alle Befunde durch eine Referenzpathologie kontrolliert. Diese erheblichen Unterschiede sind nur zu einem Teil durch Unterschiede in den Patientenkollektiven zu erklären. So liegt die Frage nahe: Wie sicher ist die Diagnose eines Carcinoma in situ der tracheobronchialen Schleimhaut? In einer kritischen Analyse fanden Venmans u. Mitarb. bedenkliche Ergebnisse im Hinblick auf die Sicherheit der Diagnose Cis. Von 45 Läsionen, die der lokale Pathologe als Carcinoma in situ befundete, wurden nur 20 durch den Referenzpathologen bestätigt $[1,11]$. Dies zeigt die Schwierigkeit und Unsicherheit, Frühveränderungen mit den vorhandenen Klassifikationskriterien korrekt einzustufen. Vor diesem Hintergrund erscheint eine eher niedrige Inzidenz plausibel. Dem entspricht die klinisch-bronchoskopische Erfahrung, dass nämlich das Carcinoma in situ einen seltenen Befund darstellt. Ähnliche Unsicherheit wie bei der Diagnose des Cis ist im Hinblick auf die präzise und reproduzierbare Klassifikation von Dysplasien dokumentiert [12].

Im Vergleich zu Carcinomata in situ sind mittel- und hochgradige Dysplasien häufige Befunde bei Risikopatienten. In den vorliegenden Studien liegt ihre Inzidenz zwischen $15[5,13]$ und 54\%
Tab. 1 Inzidenz des Carcinoma in situ in den Studien zur Autofluoreszenzbronchoskopie

\section{Inzidenz Cis}

\begin{tabular}{ll}
\hline 32/416 Biopsien $(7,7 \%)$ & Bota 2001 [6] \\
\hline 1/55 Patienten $(1,8 \%)$ & Hirsch 2001 [14] \\
0/54 Patienten $(0 \%)$ & Homasson 2001 [9] \\
10/165 Patienten $(6,1 \%)$ & Khanavkar 1998 [5] \\
\hline 9/65 Patienten $(14 \%)$ & Kusunoki 2000 [10] \\
\hline 9/700 Biopsien $(1,3 \%)$ & Lam 1998 [4] \\
\hline 19/244 Patienten (7,4\%) & Moro-Sibilot 2002 [3] \\
\hline 3/64 Patienten $(4,7 \%)$ & Shibuya 2001 [2] \\
9/1173 Patienten $(0,8 \%)$ & Häussinger 2002 [7] \\
\hline
\end{tabular}

$[4,14]$. Der zahlenmäßige Zugewinn an Information durch den Einsatz der AFB ist aufgrund der hohen Inzidenz in der Gruppe der Dysplasie-Befunde zu erwarten.

Welche klinische Bedleutung haben Dysplasie und Cis und wie groß ist das Risilko, dass aus diesen Läsionen ein invasives Karzinom entsteht?

An der Vorstellung, dass sich die Entwicklung eines invasiven Karzinoms der Lunge in mehreren Schritten vollzieht, besteht kein Zweifel. Dies ist sowohl im Hinblick auf zunehmende molekulare Alterationen als auch anhand morphologischer Kriterien gezeigt worden. Die Sequenz: gesund - metaplastisch - dysplastisch (gering-/mittel-/hochgradig) - Carcinoma in situ - invasives Karzinom wird zumindest für Plattenepithelkarzinome angenommen. Unklar ist das Risiko des Übergangs in die jeweils nächste höhere Stufe auf der Karzinomleiter.

Die publizierten Daten zum Verlauf von Dysplasie und Cis sind durch geringe Patientenzahlen und kurze Nachbeobachtungszeiten limitiert. Sie zeigen, dass beide grundsätzlich auf der Leiter der Karzinomentstehung aufsteigen können. Dysplasien können zu Cis werden und diese zu invasiven Karzinomen entarten. Bei den Verlaufsuntersuchungen in vivo ist unklar, ob bei der initialen bioptischen Materialgewinnung in Einzelfällen die dysplastische Läsion komplett entfernt wurde oder wirkliche Regredienz der Veränderung vorliegt. Dysplastische Veränderungen sind umgekehrt auch im Rahmen von Heilungsprozessen nach Biopsie vorstellbar. Für das Cis wurden spontane Remissionen, d. h. Rückgänge zur hochgradigen Dysplasie bei $22 \%$ der Läsionen gefunden $[6,15]$. Über eine Progredienz kann aus diesen Daten kein Schluss gezogen werden, da die Patienten behandelt wurden, wenn der Befund Cis persistierte. In einer kleineren Gruppe von neun beobachteten Cis entwickelten fünf (56\%) invasives Wachstum [11]. Diese Angaben weisen auf eine erhebliche maligne Potenz hin, zumal wenn man die kurze Nachbeobachtung berücksichtigt. Die hochgradige Dysplasie besitzt nach den vorliegenden Daten nur geringe Progressionstendenz. Nur 4 bzw. 11\% der Läsionen wurden im Beobachtungszeitraum zum Carcinoma in situ $[6,15]$. Dagegen zeigten ca. $63 \%$ eine dauerhafte Regredienz von der Stufe der hochgradigen zur gering- bis mittelgradigen Dysplasie. 
In einer brillianten Analyse kommen Banerjee u. Mitarb. zu dem Schluss: „es bleibt abzuwarten, ob prä-invasive Läsionen, die durch Autofluoreszenzbronchoskopie detektiert werden können, klinisch und bezüglich des Überlebens der Patienten relevant sind...“ [16]. Oder „Wird dies nur ein weiterer Beitrag sein zu der unendlich steigenden Zahl histopathologischer Präparate, die durch die Türen unserer pathologischen Institute drängen?“ [17].

Welche therapeutischen Konsequenzen zieht die Diagnose einer Dysplasie oder eines Cis nach sich?

Für die Behandlungen von hochgradigen Dysplasien oder Carcinomata in situ werden verschiedene Strategien vorgeschlagen. Von radikal-chirurgischer Intervention [18] und perkutaner Strahlentherapie [19] über Photodynamische Therapie [20], Nd:YAG-Laser-Therapie [21], Elektrokauter-Therapie [22], CryoTherapie [23], und endobronchiale Strahlentherapie (Brachytherapie) [24] bis hin zu abwartenden Strategien mit regelmäßiger bronchoskopischer Kontrolle [6]. Die Vielfältigkeit der Strategien zeigt, dass keine der Vorgehensweisen durch evidenzbasierte Daten gestützt wird. Untersuchungen zur Therapie dieser Veränderungen sind schwierig durchzuführen. Denn jede Studie zur Therapie scheitert, solange der natürliche Verlauf der Veränderungen (Dysplasie und Cis) nicht geklärt ist.

\section{Zusammenfassung}

Die Daten zur Autofluoreszenzbronchoskopie überzeugen noch nicht. Nur wenige aussagekräftige Studien belegen eine verbesserte Diagnostik von mittel- bzw. hochgradiger Dysplasie und Carcinoma in situ. Diese Veränderungen werden nur mit mäßiger Zuverlässigkeit diagnostiziert, und die therapeutischen Konsequenzen sind unklar. Es bleibt fraglich, ob die Ergebnisse spezialisierter Zentren auf alle Bronchoskopie-Einheiten übertragen werden können.

Die Autofluoreszenzbronchoskopie ist dagegen für die wissenschaftliche Arbeit über Dysplasie, Tumorentstehung und Früherkennungsmarker ein unschätzbares innovatives Instrument. Mehr optische Information mit klinisch relevanten Veränderungen zu korrelieren und klinisch nutzbar zu machen, ist die wesentliche Aufgabe. Hierzu müssen die Verbindungen zwischen dem technisch-innovativen Verfahren AFB und den modernen Möglichkeiten der Molekularbiologie enger geknüpft werden. Das Bronchialkarzinom ist wegen seiner gut evaluierten Risikofaktoren und der guten Zugänglichkeit ein geeignetes Krankheitsbild, um Tumorentstehung, -frühläsionen und -metastasierung mit Hilfe modernster Technik zu erforschen. Nur durch eine Verbesserung der Tumorfrüherkennung ist die katastrophale Prognose des Bronchialkarzinoms zu verbessern. Eine Optimierung der Patientenauswahl, z. B. durch Verbesserung von Sputumdiagnostik, und eine Verbesserung der Analyse des gewonnenen Materials, z.B. durch molekularbiologische Marker, könnte die Autofluoreszenzbronchoskopie zu einem effektiven Werkzeug in der Frühdiagnostik des Bronchialkarzinoms machen [25]. Die Autofluoreszenzbronchoskopie könnte so vom wissenschaftlichen Instrument zum Werkzeug für die tägliche klinische Arbeit avancieren.

\section{Literatur}

${ }^{1}$ Venmans BJ, Linden JC van der, Elbers JRJ et al. Observer variability in histopathological reporting of bronchial biopsy specimens: Influence on the results of autofluorescence bronchoscopy in detection of bronchial neoplasia. J Bronchol 2000; 7: 210-214

${ }^{2}$ Shibuya K, Fujisawa T, Hoshino H et al. Fluorescence bronchoscopy in the detection of preinvasive bronchial lesions in patients with sputum cytology suspicious or positive for malignancy. Lung Cancer 2001; 32: $19-25$

${ }^{3}$ Moro-Sibilot D, Jeanmart M, Lantuejoul S et al. Cigarette smoking, preinvasive lesions, and autofluorescence bronchoscopy. Chest 2002; 122: $1902-1908$

${ }^{4}$ Lam S, Kennedy T, Unger M et al. Localization of bronchial intraepithelial neoplastic lesions by fluorescence bronchoscopy. Chest 1998; 113: $696-702$

${ }^{5}$ Khanavkar B, Gnudi F, Muti A et al. Basic principles of LIFE - autofluorescence bronchoscopy. Results of 194 examinations in comparison with standard procedures for early detection of bronchial carcinomaoverview. Pneumologie 1998; 52: 71 - 76

${ }^{6}$ Bota S, Auliac JB, Paris C et al. Follow-up of bronchial precancerous lesions and carcinoma in situ using fluorescence endoscopy. Am J Respir Crit Care Med 2001; 164: 1688-1693

${ }^{7}$ Häussinger K. European D-LIGHT Experience. 12th World Congress for Bronchology. Boston, USA, 2002

${ }^{8}$ Stanzel F. Results of the D-Light study. ERS-Coference. Wien, Österreich, 2003

${ }^{9}$ Homasson JP, Capron F, Angebault M et al. Lung autofluorescence. Preliminary study of two systems without laser illumination or photosensitization. Rev Pneumol Clin 2001; 57: 202 - 207

${ }^{10}$ Kusunoki Y, Imamura F, Uda $\mathrm{H}$ et al. Early detection of lung cancer with laser-induced fluorescence endoscopy and spectrofluorometry. Chest 2000; 118: 1776-1782

${ }^{11}$ Venmans BJW, Boxem TJM van, Smit EF et al. Outcome of Bronchial Carcinoma in situ. Chest 2000; 117: 1572-1576

${ }^{12}$ Nicholson AG, Perry LJ, Cury PM et al. Reproducibility of the WHO/ IASLC grading system for pre-invasive squamous lesions of the bronchus: a study of inter-observer and intra-observer variation. Histopathology 2001; 38: 202-208

${ }^{13}$ Kurie JM, Lee JS, Morice RC et al. Autofluorescence bronchoscopy in the detection of squamous metaplasia and dysplasia in current and former smokers. J Natl Cancer Inst 1998; 90: 991 - 995

${ }^{14}$ Hirsch FR, Franklin WA, Gazdar AF et al. Early Detection of Lung Cancer: Clinical Perspectives of Recent Advances in Biology and Radiology. Clin Cancer Res 2001; 7: 5-22

15 Thiberville L, Metayer J, Raspaud C et al. A prospective, short-term follow up study of 59 severe dysplasias and carcinoma in situ of the bronchus using autofluorescence endoscopy. Eur Respir J Suppl 1997; 10: $425 \mathrm{~s}$

${ }^{16}$ Banerjee AK, Rabbitts PH, George J. Lung cancer 3: Fluorescence bronchoscopy: clinical dilemmas and research opportunities. Thorax 2003; 58: 266-271

${ }^{17}$ Kerr KM. Pulmonary preinvasive neoplasia. J Clin Pathol 2001; 54: $257-271$

18 Saito Y, Nagamoto N, Ota S et al. Results of surgical treatment for roentgenographically occult bronchogenic squamous cell carcinoma. J Thorac Cardiovasc Surg 1992 Aug; 104 (2): 401 - 407

19 Gauden S, Ramsay J, Tripcony L. The curative treatment by radiotherapy alone of stage I non-small cell carcinoma of the lung. Chest 1995 Nov; 108 (5): $1278-1282$

${ }^{20}$ Kato H. Photodynamic therapy for lung cancer - a review of 19 years' experience. J Photochem Photobiol B 1998 Feb; 42 (2): 96 - 99 Review

${ }^{21}$ Boxem AJ van, Westerga J, Venmans BJ et al. Photodynamic therapy, Nd-YAG laser and electrocautery for treating early-stage intraluminal cancer: which to choose? Lung Cancer 2001 Jan; 31 (1): 31 - 36

22 Boxem TJ van, Venmans BJ, Schramel FM et al. Radiographically occult lung cancer treated with fibreoptic bronchoscopic electrocautery: a pilot study of a simple and inexpensive technique. Eur Respir J 1998 Jan; 11 (1): 169-172

23 Thurer RJ. Cryotherapy in early lung cancer. Chest 2001 Jul; 120 (1): 3 - 5

${ }^{24}$ Perol M, Caliandro R, Pommier P et al. Curative irradiation of limited endobronchial carcinomas with high-dose rate brachytherapy. Results of a pilot study. Chest 1997 May; 111 (5): 1417-1423

${ }^{25}$ Kennedy TC, Lam S, Hirsch FR. Review of recent advances in early localization of central airway lung cancer. Oncologist 2001; 6: 257-262 